\title{
Hempelian and Kuhnian Approaches in the Philosophy of Medicine: the Semmelweis Case
}

\author{
Donald Gillies \\ Department of Science and Technology Studies, University College London, Gower \\ Street, London WC1E 6BT. Email: donald.gillies@ucl.ac.uk
}

\section{Introduction}

2. Semmelweis's Investigations

3. Semmelweis's Failure

4. Kuhn's Ideas applied to the Semmelweis Case

5. Strengths and Limitations of a Kuhnian Approach

\section{Introduction}

It is a rather surprising fact that medicine was largely ignored by the majority of philosophers of science in the twentieth century, despite the fact that medicine made enormous progress in the last century. A list of famous philosophers of science of the twentieth century would surely include Carnap, Duhem, Feyerabend, Kuhn, Lakatos, Neurath, Poincaré, Popper, Quine, but none of these have discussed in detail an example taken from medicine. Naturally, however, there are some notable exceptions to this general rule. One of these is Carl Hempel. In his book: Philosophy of Natural Science, published in 1966, Hempel's very first example of an admirable scientific investigation is taken from the history of medicine. It is the work of Ignaz Semmelweis in Vienna in the $19^{\text {th }}$ Century. Hempel's choice was an excellent one. The example is not too technical, but it yet deals with an episode which is very interesting and important for the history of medicine. For these reasons, I will follow Hempel in analysing this example. In section 2 I will describe Semmelweis's investigations, and consider Hempel's treatment of these. My thesis will be that there are certain aspects of the Semmelweis case which are not adequately explained by Hempel's analysis. In particular that analysis does not explain why Semmelweis did not succeed in persuading the medical community that his new ideas were correct. In the next section (3), I will describe this failure of Semmelweis, and discuss possible reasons why it happened. Then in section 4 I will argue that Kuhn's ideas applied to the Semmelweis case do shed some light on why Semmelweis failed. Finally in section 5, I will consider the strengths and limitations of such a Kuhnian approach. Let us now turn to considering Semmelweis and his scientific work.

\section{Semmelweis's Investigations}

Ignaz Semmelweis (1818-65) was Hungarian. He was born in Taban (now part of Budapest) in July 1818, the son of a prosperous grocer. Hungary was then part of the Austrian empire, whose capital was Vienna. Semmelweis went to the University of 
Vienna in 1837. He originally intended to study law, but switched to medicine. He qualified in March 1844 and obtained the degree of Master of Midwifery in August 1844. For the next two years he held the rather humble post of aspirant to assistant at the first maternity clinic, and he then became full assistant in 1846. As the exact dates when Semmelweis held this post of assistant are important in what follows, it is worth quoting what he himself says on this subject in the preface to his book (Semmelweis, 1861, p. 61):

'On 1 July 1846 I officially assumed the position of assistant at the first maternity clinic. On 20 October of the same year, however, I was obliged to withdraw in favor of my predecessor, Dr. [Franz] Breit, because Dr. Breit had in the meantime received a two-year extension of his service. That I may be understood, in the course of this essay I will refer to these four months, namely July, August September, and October of 1846, as my first period of service. ... Then Dr. Breit was named Professor of Obstetrics at the medical school at Tübingen, and for the second time I officially assumed the position of assistant, on 20 March 1847. I functioned as such for two years, until 20 March 1849. I refer to these two years as my second period of service.'

The General Hospital of Vienna contained a Lying-In or Maternity Hospital as part. This was enormous by contemporary standards. By the $1850 \mathrm{~s}$ and $1860 \mathrm{~s}$ it catered for about 7,000 to 8,000 patients a year compared with around 200 to 300 in the General Lying-In Hospital in London (Loudon, 1992, p. 65). When Semmelweis began his career, one of the worst risks of childbirth was contracting a terrible disease, now fortunately eliminated, called puerperal fever because it normally struck during the puerperium, or approximately 6 weeks after childbirth when the womb returns to its normal shape. Loudon describes how the disease could strike as follows (1992, p. 54):

'A woman could be delivered on Monday, happy and well with her newborn baby on Tuesday, feverish and ill by Wednesday evening, delirious and in agony with peritonitis on Thursday, and dead on Friday or Saturday.'

The Vienna Maternity Hospital was divided into two clinics from 1833. Patients were admitted to the two clinics on alternate days thereby (as Loudon points out, 1992, p. 65): 'producing, unintentionally, a system of random allocation.' Between 1833 and 1840, medical students, doctors and midwives attended both clinics, but, thereafter, although doctors went to both clinics, the first clinic only was used for the instruction of medical students who were all male in those days, and the second clinic was reserved for the instruction of midwives. When Semmelweis began working as an assistant in 1846, the mortality statistics showed a strange phenomenon (see Table 1).

\section{$\underline{\text { Table } 1}$}

Between 1833 and 1840, the death rates in the two clinics had been comparable, but, in the period 1841-46, the death rate in the first clinic was $9.92 \%$ and in the second clinic $3.88 \%$. The first figure is more than 2.5 times the second - a difference which is certainly 
statistically significant. The quoted figures actually underestimate the difference since some severe cases of puerperal fever were removed from the first clinic to the general hospital where they died - thereby disappearing from the first clinic's mortality statistics. This rarely happened in the second clinic (Semmelweis, 1861, pp. 64-5). Semmelweis was puzzled and set himself the task of finding the cause of the higher death rate in the first clinic.

Let us now return to Carl Hempel and his 1966 book: Philosophy of Natural Science. As his very first example of an excellent scientific enquiry, Hempel chooses Semmelweis's work on childbed fever. Semmelweis's investigation very much follows the Popperian model of conjectures and refutations. Semmelweis considers various hypotheses in turn. He rejects those which are contradicted by the facts, and eventually comes up with one which agrees with the observations. This hypothesis is then further confirmed by a practical test. Let us now turn to details. ${ }^{1}$

The first hypothesis considered by Semmelweis (1861, pp. 65-66) was that the higher death rate in the first clinic was due to 'atmospheric-cosmic-terrestial' factors. This sounds strange but is just a way of referring to the miasma theory of disease which was standard at the time. According to this theory, disease was due to a putrid atmosphere or miasma. There was much evidence to support this theory, since, for example, malaria occurred in marshlands, and diseases of all kinds were more common in overcrowded slums, barracks, ships and workhouses where the atmosphere often was evil smelling. Moreover the miasma theory did lead to valuable reforms. It was held by Chadwick who pressed for improved sewage disposal, drainage, cleaning and sanitary regulation of buildings in Britain in the 1840s (Porter, 1997, p. 411). In the hospitals it led to a belief in cleanliness, fresh air, avoidance of overcrowding, etc. Florence Nightingale was another believer in the miasma theory. However Semmelweis pointed out that it could not explain the different mortality rates in the first and second clinics. These were under the same roof and had an ante-room in common. So they must be exposed to the same 'atmospheric-cosmic-terrestial' influences. Yet the death rates in the two clinics were very different.

The next hypothesis was that overcrowding was the key factor, but this too was easily refuted since the second clinic was always more crowded than the first, which, not surprisingly had acquired an evil reputation among the patients, almost all of whom tried to avoid it (Semmelweis, 1861, p. 69).

As the situation had obviously become critical, a commission was appointed to look into it, and, at the end of 1846, it came up with the theory that the medical students in the first clinic, especially the foreign students, were examining the patients in a rougher manner than the student midwives of the second clinic. Semmelweis $(1861$, p. 84) describes the consequences as follows:

'As a result of this opinion the number of students was reduced from forty-two to twenty. Foreigners were almost entirely excluded, and examinations were reduced to a minimum. The mortality rate did decline significantly in December 1846, and in January, February, and March of 1847. But in spite of these measures, fifty-seven patients died in April and thirty-six more in May. This demonstrated to everyone that the view was groundless.' [See Table 2 for detailed figures - D.G.] 
In this sort of way Semmelweis eliminated quite a number of curious hypotheses. One concerned the appearance of a priest to give the last sacrament to a dying woman. The arrangement of the rooms meant that the priest, arrayed in his robes, and with an attendant before him ringing a bell had to pass through 5 wards of the $1^{\text {st }}$ clinic before reaching the sickroom where the woman lay dying. The priest had, however, direct access to the sickroom in the case of the $2^{\text {nd }}$ clinic. The hypothesis then was that the terrifying psychological effect of the priest's appearance debilitated patients in the first clinic, and made them more liable to puerperal fever. Semmelweis recounts how he tested this hypothesis as follows (1861, pp. 71-72):

'During my first period of service, I appealed to the compassion of the servant of God and arranged for him to come by a less direct route, without bells, and without passing through the other clinic rooms. Thus, no one outside the room containing the ill patients knew of the priest's presence. The two clinics were made identical in this respect as well, but the mortality rate was unaffected.'

I won't mention any more of the hypotheses which Semmelweis was able to test and refute, but I will say something about another factor which influenced his thinking. There were in Vienna at the time many street births, and yet, despite what would seem to be maximally unfavourable conditions, the mothers were usually free from puerperal fever. The evidence here was rather anecdotal, and when Skoda, a friend of Semmelweis, asked for the collection of statistics, a commission was named to look into the matter. But as a result of protests from the professor in charge of the clinics, Johann Klein, the commission never began its activity. Professor Klein, according to Sinclair (1909, p. 43): 'in fact obtained the interference of the Minister of Education to prevent investigation.'

After trying out all these hypotheses unsuccessfully, Semmelweis was in a depressed state in the winter of 1846-7. However a tragic event early in 1847 led him to formulate a new hypothesis. On $20^{\text {th }}$ March 1847 , Semmelweis heard with sorrow of the death of Professor Kolletschka. In the course of a post-mortem examination, Professor Kolletschka had received a wound on his finger from the knife of one of the students helping to carry out the autopsy. As a result Kolletschka died not long afterwards of a disease very similar to puerperal fever. Semmelweis reasoned that Kolletschka's death had been owing to cadaverous matter entering his bloodstream. Could the same cause explain the higher death rate of patients in the first clinic? In fact professors, assistants and students often went directly from dissecting corpses to examining patients in the first clinic. It is true that they washed their hands with soap and water, but perhaps some cadaverous particles still adhered to their hands. Indeed this seemed probable since their hands often retained a cadaverous odour after washing. The doctors and medical students might then infect some of the patients in the first clinic with these cadaverous particles, thereby giving them puerperal fever. This would explain why the death rate was lower in the second clinic, since the student midwives did not carry out post-mortems. It would also explain why those giving birth in the street would escape puerperal fever despite the unfavourable conditions.

In order to test this hypothesis, Semmelweis from some time in May 1847 required everyone to wash their hands in disinfectant before making examinations. At 
first he used chlorina liquida, but, as this was rather expensive, chlorinated lime was substituted. The result was dramatic. In 1848 the mortality rate in the first clinic fell to $1.27 \%$, while that in the second clinic was $1.30 \%$. For this, and further statistics, see tables 1 and 2.

Two further incidents led Semmelweis to extend his hypothesis. The first (the incident of the medullary carcinoma), he describes as follows (Semmelweis, 1861, p. 93):

'In October 1847, a patient was admitted with discharging medullary carcinoma [cancer of the innermost part] of the uterus. She was assigned the bed at which the rounds were always initiated. After examining this patient, those conducting the examination washed their hands with soap only. The consequence was that of twelve patients then delivering, eleven died. The ichor [foul-smelling watery discharge from a wound, ulcer, or diseased organ - D.G.] from the discharging medullary carcinoma was not destroyed by soap and water. In the examinations, ichor was transferred to the remaining patients, and so childbed fever multiplied. Thus, childbed fever is caused not only by cadaverous particles adhering to hands but also by ichor from living organisms. It is necessary to clean the hands with chlorine water, not only when one has been handling cadavers but also after examinations in which the hands could become contaminated with ichor. This rule, originating from this tragic experience, was followed thereafter. Childbed fever was no longer spread by ichor carried on the hands of examiners from one patient to another.'

The second incident was that of the carious knee, and is described as follows (Semmelweis, 1861, p. 93):

'A new tragic experience persuaded me that air could also carry decaying organic matter. In November of the same year, an individual was admitted with a discharging carious left knee. In the genital region this person was completely healthy. Thus the examiners' hands presented no danger to the other patients. But the ichorous exhalations of the carious knee completely saturated the air of her ward. In this way the other patients were exposed and nearly all the patients in that room died. The reports of the first clinic indicate that eleven patients died in November and eight more in December [See Table 2 - D.G.]. These deaths were largely due to the ichorous exhalations from this individual. The ichorous particles that saturated the air of the maternity ward penetrated the uteruses already lacerated in the birth process. The particles were resorbed, and childbed fever resulted. Thereafter, such individuals were isolated to prevent similar tragedies.'

We see from this that Semmelweis introduced his antiseptic precautions in two stages. Chlorine washings after carrying out dissections began sometime in May 1847, and, as we see from table 2 , these reduced the death rate in the first clinic for the rest of the year to $3.04 \%$. The further precautions of chlorine washings after examining patients with an infection, and of isolating patients with an infection which might pollute the atmosphere, were introduced at the end of 1847. In 1848 the death rate in the first clinic was reduced still further to $1.27 \%$ - actually less than that of the second clinic $(1.30 \%)$. This was the first time the mortality rate in the first clinic had been lower than that of the second clinic since the medical students had been divided from the student midwives. Chlorine washings seem to have been continued in the Vienna Maternity Hospital even 
after Semmelweis's departure, and the death rates in the two clinics remained about the same for the next decade, as is shown in table 1. It is interesting to note that the lowest death rate in the first clinic occurred in 1848 which was the only year in which Semmelweis was in charge and his full set of antiseptic precautions had been devised. This could, however, be a statistical fluctuation.

One other interesting feature of table 2 is the sudden dramatic increase in the death rate in the first clinic in the months of April and May 1847. From an average of $2.91 \%$ in January to March it rises to $15.35 \%$ in April to May. With remarkable honesty Semmelweis declares that he himself was responsible, unwittingly of course, for this increase. It will be recalled that Semmelweis's predecessor as assistant to the first clinic was Dr Breit, and that Semmelweis took over from him on 20 March 1847. It seems that Dr Breit was not very industrious when it came to carrying out dissections and during his time as assistant the students followed his example. This explains the low death rate in the period January to March 1847. When the energetic Semmelweis took over, however, dissections were resumed on a grand scale and the students no doubt followed the example of their industrious teacher. As a result the death rate shot up in April and May. This is a curious example of hard work and conscientiousness producing much worse results. Semmelweis himself describes it as follows (1861, pp. 102-3):

'... the assistant of the first clinic seldom visited the morgue in the months of ... January, February, and March 1847. The Austrian students, whose number was reduced to eighteen, followed his example. The opportunity for them to contaminate their hands with cadaverous particles was thereby greatly reduced. Restricting examinations to the minimum also reduced the opportunity for the genitals of patients to be touched by contaminated hands. For these reasons, mortality in the first clinic was reduced during these months.

On 20 March 1847, I reassumed the position of assistant in the first clinic. Early that morning I conducted my gynecological studies in the morgue. I then went to the labor room and began to examine all the patients, as my predecessors and I were obliged to do, so that I could report on each patient during the professor's morning rounds. My hands, contaminated by cadaverous particles, were thereby brought into contact with the genitals of so many women in labor that in April, from 312 deliveries, there were 57 deaths ... . In May, from 294 deliveries there were 36 deaths ... .'

The commission of 1846 which had met to consider the problem of the high death rate in the first clinic had recommended that foreigners be excluded. This certainly seems an absurd example of national prejudice. Yet Semmelweis argued that there was truth in it - not because the foreign students were worse in any respect but rather because they were on average more conscientious and hard-working. This is how he puts it (1861, p. 104):

'Everyone, even those who do not share my opinion, will agree that the commission acted groundlessly in imputing guilt to foreigners. In fact, I alone held that foreigners were more dangerous than natives, but not because they examined more roughly. The reason that foreigners were more dangerous than natives lies in the following considerations.

Foreigners come to Vienna to perfect medical training already begun in their own universities. ... they utilize their time as efficiently and educationally as possible. They 
have, therefore, many opportunities for their hands to become contaminated with foul animal-organic matter. Thus, it is no wonder that foreigners, busy in the maternity hospital at the same time, are more dangerous for patients.'

To sum up then. As a result of his scientific investigations in the years 1846-7, Semmelweis reached the conclusion that puerperal fever is caused by cadaverous particles or other decaying matter derived from living organisms being conveyed to the patients. It is conveyed by the hands of the doctors, or, in some cases, by the air. Puerperal fever can therefore be prevented by the doctors washing their hands in disinfectant before examinations, and by isolating patients whose condition might infect the atmosphere. Hempel, as we have seen, mentions Semmelweis's work as his very first example of a really excellent scientific investigation, but Hempel does not mention one curious fact. Semmelweis's theory was not accepted by his fellow doctors. In the next section I will give an account of Semmelweis's failure to convince the medical profession of his new ideas. Hempel's analysis of the Semmelweis case does not explain this failure and indeed makes it more mysterious. However, I will argue that the introduction of Kuhnian ideas can remedy this defect in Hempel's account.

My main criticism of Hempel is then that his account is incomplete rather than that it is inadequate. This contrasts with a discussion of Hempel and Semmelweis by Lipton in his 1991 (pp. 75-98). Lipton takes issue with Hempel's hypothetic-deductive account, and tries to show (p. 88): "that Inference to the Best Explanation is better than hypothetico-deductivism." Lipton's main argument against Hempel's hyptheticodeductivism is the following (pp. 91-2):

'According to him [Hempel -D.G.] the hypotheses that appealed to overcrowding, diet, or general care were rejected because the claims that the difference in mortality between the divisions was due to such differences 'conflict with readily observable facts' $(1966$, p. 6). The claim that, for example, the difference in mortality is due to a difference in diet is incompatible with the observation that there is no difference in diet. These are clearly cases of logical incompatibility, but they are not the ones Hempel needs: the claims that are incompatible with observation are not the general hypotheses Semmelweis rejects. ... the hypotheses of overcrowding, diet, and care are surely general conjectures about causes of childbed fever, not specific claims about the differences between the divisions. But the hypotheses that overcrowding, diet, or general care is a cause of childbed fever are logically compatible with everything Semmelweis observes."

Now Semmelweis does begin by considering hypotheses as to the cause of the difference in death rates between the two clinics, and these can certainly be refuted by observation as Semmelweis points out. Yet Lipton is right in that Semmelweis moves on later to consider more general hypotheses about causes of childbed fever. Indeed he proposes such a general hypothesis himself. However in moving on to these more general hypotheses, Semmelweis continues to regard hypotheses such as overcrowding as having been refuted. There seems, as Lipton points out, to be a gap here in his logic from the point of view of the hypothetico-deductive account. After all overcrowding, though it could not be the cause of the higher mortality in the first clinic might nonetheless be $\underline{a}$ cause of puerperal fever. Different cases of puerperal fever might have had different 
causes, or a particular case might have been brought about by a combination of various causes. Why then does Semmelweis, having shown that overcrowding cannot be the cause of the higher mortality rate in the first clinic, not consider that it might still be a cause of puerperal fever is some cases? The reason, I think, is that Semmelweis made the assumption that puerperal fever has a single necessary cause, so that having shown that cadaverous particles cause the difference in mortality rates between the two clinics, he concludes that the cause of puerperal fever in general must be something of which the cadaverous particles are a special case. This rules out overcrowding as a cause. I will consider Semmelweis's attitude to causality in medicine further in section 5, where it will be shown that it was unusual for that time. However, let us now turn to examining the reception which Semmelweis's new theory received.

\section{Semmelweis's Failure}

Semmelweis's two year post as assistant came to an end on 20 March 1849. He applied for an extension for a further two years, but this was not granted. He then applied for the position of Privat-Dozent of Midwifery, but this was not granted. He applied again to become Privat-Dozent in February 1850. He was made Privat-Dozent in October, but with some humiliating restrictions on what he was allowed to do. Disgusted with his treatment, he left for his native Hungary without even saying good-bye to his friends. Thereafter he remained in Budapest largely ignored and forgotten. In 1865 he became mad, and had to be committed to an asylum in Vienna, where he died the same year from an infected finger. It used to be thought that Semmelweis's madness was brought on by the rejection of his ideas by his colleagues, but a recent examination of his symptoms suggests that he may have had premature Alzheimer's. He was only 47.

The year in which Semmelweis died (1865) was, oddly enough, the year in which Lister first tried out his antiseptic system. Lister was later asked whether he had been influenced by Semmelweis, but said that he had not heard of Semmelweis at that time, and that even when he visited Budapest 20 years later in 1885, he still heard nothing from the doctors who entertained him there of Semmelweis whose "name was never mentioned, having been, as it seems, as entirely forgotten in his native city as in the world at large.' (From a letter of Lister's 15 September 1906 cited in Godlee, 1917, 141).

Codell Carter does however point out that Lister could have come across Semmelweis's work (1983, p. 54). Thus Lister might have been influenced by Semmelweis either unconsciously or indirectly. However the connection is very tenuous.

Semmelweis advocated antisepsis in obstetrics, and Lister in surgery. Yet the fate of the two men was very strikingly different. Whereas Semmelweis failed to get his new methods generally accepted, Lister succeeded. Whereas Semmelweis was ignored and forgotten, Lister became President of the Royal Society (1895), the first medical man to gain a peerage (1897), and was showered with honours both nationally and internationally. Why was there such a striking difference? To answer this question, let us begin by looking in more detail at the reasons for Semmelweis's failure.

One reason why Semmelweis's theory might have been disliked was that it suggested that the doctors themselves were the principal cause of puerperal fever by bringing decaying matter to the patients in their examinations. Such a view, it could be argued, was unlikely to appeal to the doctors. 
A second possible reason was that Semmelweis, though excellent in practical work and investigations, seems to have had a block about writing up and publishing his results. He actually speaks in the Preface to his main work (Semmelweis, 1861, p. 62) of 'my innate aversion to every form of writing.' The first account of his work was actually published by his friend Hebra in an article of December 1847, followed by a second article. Semmelweis was also invited to address the Vienna Medical Society in 1849, but he refused to do so, and the talk was given by his friend Skoda instead. In fact it was not until 1861 that Semmelweis published an account of his work. This was in a book: The Etiology, Concept and Prophylaxis of Childbed Fever of 543 pages. We have already quoted extensively from this book which is certainly a classic of the history of medicine. Semmelweis presents a vivid account of his investigations, states his views clearly, and often develops incisive arguments in their favour. Yet this classic is a flawed one. Semmelweis is very repetitious, and has other faults besides. Semmelweis cannot refrain from praising himself. He speaks (1861, p. 214) of 'the eternal truths I have discovered', and says (pp. 213-14):

'... with the exception of Jenner's cowpox inoculations, there is no third discovery in the whole history of medicine that, by protecting against a disease, can save as many human lives as my teaching regarding protection against childbed fever.'

Semmelweis also compares his own successes in reducing the death rate from puerperal fever with the relative failures of his contemporaries who held different theories from him. Thus he says $(1861,224)$ :

'... puerperal fever is very much dependent on the theories of physicians. Dr. Lumpe explains the origin of puerperal fever by epidemic influences and he sends nearly one patient to the morgue every day. I explain the origin of puerperal fever by infection with decaying matter and in 1848 I sent only forty-five patients to the morgue.'

Generally speaking when Semmelweis is discussing the views of his opponents, he treats them in a harsh, polemically, and indeed scornful fashion. Thus, for example, he says (1861, pp. 232-3):

' ... 823 of my students are now midwives practicing in Hungary. ... They are more enlightened than the members of the Society for Obstetrics in Berlin; they would laugh Virchow to scorn if he attempted to lecture them on epidemic puerperal fever.'

Virchow was one of the leading German medical researchers of the time.

Apart from all this, the sheer length of Semmelweis's book must have been a disincentive to reading it. If Semmelweis had cut down the size of his book to say 150 pages, and confined himself to stating his own views and not discussing those of his contemporaries, then his book might have had an impact. In the event it was ignored. Altogether Semmelweis was not very good at promoting his own views.

A third possible reason for Semmelweis's failure concerns his nationality and academic position. He was Hungarian, and, within the Austrian empire at that time, Hungarians were often regarded as inferior by the dominant Austrians. Moreover, Semmelweis was only a lowly temporary assistant while his ideas were opposed by the 
Professor and Head of the Clinics, Johann Klein. Klein was not only a professor in a superior position, but a native Austrian. In the authoritarian German-speaking academic world of the time, so it could be argued, it would have been very unlikely the views of a humble Hungarian temporary assistant would have been preferred to those of an Austrian Herr Professor.

The three 'external' reasons just described probably did have a certain importance, but they are not, in my view, quite as important as they might seem at first sight. I will argue for this claim in section 5, but will now introduce an 'internal' reason which I think is of greater importance.

A key point here is that some practices, such as antisepsis, need a theoretical justification. Semmelweis's recommendation that doctors and medical students washed their hands in chlorinated lime before examinations did indeed produce a change in mortality statistics, but this could always be dismissed as a statistical fluctuation. After all, there was considerable variation in death rates anyway. The washing process only made sense, and probably would only have been carried out properly, by those who believed Semmelweis's theory that the cause of puerperal fever was cadaverous particles, or other decaying matter derived from living organisms, being conveyed to the patient. Semmelweis did believe this theory, but it was not accepted by the majority of the medical profession at the time. But why was a theory, which now seems so very reasonable, not acceptable at that time? To answer this question, we must introduce the ideas of Kuhn, and I will do so in the next section. ${ }^{2}$

\section{Kuhn's Ideas applied to the Semmelweis Case}

To introduce Kuhnian themes, I will begin by pointing out an inadequacy in Hempel's account of scientific method. Hempel's account is in fact quite Popperian. Conjectures are proposed and tested out. Most of them are refuted, but a fortunate scientist may eventually hit on a conjecture which agrees with the facts, passes tests, and so may be tentatively adopted for a while. In the light of Kuhn's ideas, however, such an account must be qualified. A Kuhnian would argue that during a period of normal science, a scientist cannot put forward any arbitrary conjecture (as Popper suggests) but only a conjecture which is compatible with the dominant paradigm. If a scientist puts forward a conjecture which contradicts the dominant paradigm, it is likely to be dismissed out of hand as absurd. Admittedly in some cases, e.g. Copernicus, such a hypothesis may mark the beginning of a revolution, but, even if the hypothesis is vindicated in the long run, it is likely to be strongly opposed at first by the scientific community.

This suggests a Kuhnian reason for Semmelweis's failure. ${ }^{3}$ His practice needed to be justified by a theory, and the theory he put forward was rejected because it contradicted the then dominant paradigm concerning the causation of disease. Lister's antiseptic system was, by contrast, accepted because its introduction coincided with a revolutionary period in medical theory in which this older paradigm was replaced by the germ theory of disease. Let us now examine in more detail whether this Kuhnian model fits the historical facts.

If we look at the theories of the causation of disease which were held in Semmelweis's day, it is not immediately clear whether we should classify these in Kuhn's 
terminology as constituting a paradigm, or as forming part of a pre-paradigmatic period. There were two principal theories - the miasma theory, which we have already described, and the contagion theory. The existence of two different theories suggests a preparadigmatic period. Kuhn illustrates his concept of a pre-paradigmatic period with the example of theories of light. According to Kuhn, the first paradigm in the study of light was Newton's particle theory which came to be generally accepted in the early eighteenth century. Before there had been different schools of thought each of which held a different view as to the nature of light and so no general consensus. The existence of these different schools of thought constitutes for Kuhn a pre-paradigmatic period. As he says (1962, p. 11):

'No period between remote antiquity and the end of the seventeenth century exhibited a single generally accepted view about the nature of light. Instead there were a number of competing schools and sub-schools, most of them espousing one variant or another of Epicurean, Aristotelian, or Platonic theory. One group took light to be particles emanating from material bodies; for another it was a modification of the medium that intervened between the body and the eye; still another explained light in terms of an interaction of the medium with an emanation from the eye; and there were other combinations and modifications besides.'

Such was the situation in the pre-paradigmatic period in the study of light. However, closer consideration shows that the situation in medicine in the 1840 s was not at all similar. It is true that there were two dominant theories - the miasma theory and the contagion theory; but these theories were not held by contending schools of thought in medicine. On the contrary, almost all doctors held that both theories were correct and that diseases were to be explained in some cases by one of the theories, in other cases by the other theory, and in still other cases by some combination of the two theories. What we have then is not a pre-paradigmatic period, but rather what could be described as a composite paradigm.

To clarify the nature of this composite paradigm, it is useful to look at Jacob Henle's classic 1840 paper: 'On Miasmata and Contagia'. At first sight this may seem an odd choice since this paper is famous as one of the forerunners of the germ theory of disease. Indeed Henle does argue for the idea of a contagium vivum, but at the same time he regarded this as quite compatible with the miasma and contagion theories. For him, the contagium vivum merely supplied a deeper explanation of these theories. As we shall see in a moment, Semmelweis's attitude was very different.

Because of his 'compatibilist' attitude, Henle can be taken as a good exponent of the dominant miasma and contagion theories of his day. His general approach is to explain diseases in terms of either one of the two theories, or of a combination of the two theories. This is how he puts it (1840, pp. 913-4):

'... I wish ... to divide the endemic and epidemic diseases into three groups. The first consists of those which are only miasmatic and, as far as one knows, are never contagious. I include only the ague [i.e. malaria-D.G.] in this group. In the second group belong the diseases, which appear miasmatically, but apparently are also spread through contagions; such are the contagious exanthemata, small-pox, measles, rubella, scarlet fever; in addition typhoid, certain types of cold and catarrh, particularly influenza, 
dysentery, cholera, plague, and one form of puerperal fever. ... In the third group finally, I wish to place those diseases which arise only through contagions, and which, nowadays at least, are not seen to develop miasmatically; such are syphilis, scabies ...'

What we see here is an attempt to classify diseases into various classes, and then to explain the diseases of a particular class in terms of a theory or combination of theories. This is quite characteristic of medicine, but much less so of physics and chemistry. Now Kuhn's concept of paradigm was largely based on examples taken from physics and chemistry. If we are to extend it to medicine, as I think we should, it needs to be modified somewhat.

To see what is needed, let us take a typical Kuhnian paradigm, namely the Newtonian paradigm of $18^{\text {th }}$ and $19^{\text {th }}$ century physics. The core of this paradigm consisted of Newton's 3 laws of motion and his law of gravity. These laws were held to apply to all bodies in the universe. Now certainly in medicine we are never going to have this kind of generality. The expression: 'the germ theory of disease' suggests that it was meant to be a theory of all diseases, but this was not the case. The theory was not intended to apply to many congenital diseases or cancers. In fact a paradigm for medicine at a given moment is composite in nature, and consists of (i) a general classification scheme for diseases, and (ii) a number of local paradigms applying to the diseases within a given class.

The composite nature of paradigms in medicine as opposed to the simpler paradigms of physics gives rise to phenomena in the development of medicine which are not to be found in the history of physics and which are not considered by Kuhn. In a Kuhnian revolution, an old paradigm is overthrown and replaced by a new one. In medicine, however, it is possible to have very important changes which do not have this character, but consist in the reclassification of a disease. This may result in a complete change in the explanation of what causes the disease and in the recommended therapy without however introducing any new local paradigms. An excellent example of such a development in medicine is the change from the excess acid theory to the bacterial theory of peptic ulcers, which Thagard describes and analyses in his 1999, pp. 39-97.

The change to the bacterial theory of peptic ulcers was brought about by the work of two Australian scientists: Robin Warren and Barry Marshall. Before their innovation, peptic ulcers had been thought to be caused by excessive stomach acidity. ${ }^{4}$ This in turn was thought to be often the result of stress, since experiments on animals showed that stress did produce higher concentrations of stomach acid. We can call this: 'the excess acid theory'. It naturally led to treating peptic ulcers by anti-acid drugs which, although they provided relief, did not in general succeed in eliminating the ulcers or preventing their recurrence. It was further thought that no bacteria could survive for long in the human stomach because of its high acid concentration. This effectively ruled out a bacterial theory of ulcers.

The first surprise came in 1979 when Warren observed spiral bacteria in a biopsy specimen from a human stomach. In 1981 Marshall joined Warren to help him investigate the new bacterium. At first it was thought to belong to the Campylobacter genus, but further investigation showed this to be false and that the genus was a new one which was named Helicobacter. The specific bacterium was eventually called

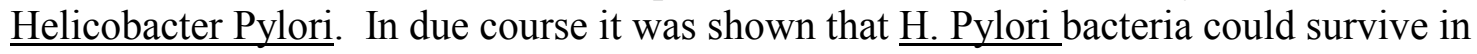
the stomach by burying themselves beneath the mucosal layer and producing ammonia to 
neutralise the gastric acid.

Warren and Marshall went on to suggest that their new discovered stomach bacteria might be the cause of peptic ulcers. When this suggestion was first published in 1983 , it was greeted by widespread scepticism because it so obviously contradicted accepted accounts of the disease. However further testing and investigation led to more and more evidence in favour of the new hypothesis, and by 1995 it had come to be generally accepted. This led in turn to treating peptic ulcers with antibiotics, though it was discovered that a combination of these with some of the old anti-acid drugs provided the best therapy.

The change from the excess acid theory to the bacterial theory of peptic ulcers can be considered as a 'mini-revolution' in medicine. However it is important to note how it differs from some of the classic revolutions in physics or chemistry considered by Kuhn. Take for example the chemical revolution. Before this revolution, the phlogiston theory had been used to explain a range of phenomena, such as combustion and calcination, as brought about by the release of phlogiston. After the revolution, the same phenomena were explained as caused by the addition of oxygen. The new oxygen paradigm which emerged was based on mechanisms which had not been considered before and were newly devised. By contrast the new bacteria theory of ulcers was based on a mechanism (infection by bacteria) which had been well-established for nearly a hundred years. The 'mini-revolution' consisted of a reclassification of the disease ${ }^{5}$ without introducing any new local paradigms. This is a good illustration of how the composite paradigms of medicine consisting of a classification scheme for diseases together with a local paradigm for the diseases of each class differ from the simpler paradigms considered by Kuhn.

Returning to the Semmelweis case, my conclusion is that we can legitimately speak of a dominant paradigm in medicine in the 1840s. This consisted of a classification of diseases and their explanation in terms of the miasma and contagion theories, either separately or in combination. Of course this does not give a complete picture of the medical ideas then current, but it is sufficient to deal with the problem of the reception of Semmelweis's theory. In fact I will now argue that Semmelweis's views contradicted the dominant medical paradigm of his time.

Generally speaking, Semmelweis's hypothesis regarding the causation of puerperal fever contradicted both the miasma theory and the contagion theory. This statement needs a slight qualification since one part of Semmelweis's theory (the incident of the carious knee) and the recommendations based on it agree with the miasma theory. However, the rest of Semmelweis's theory clearly contradicts the miasma theory. At first sight it might be thought that Semmelweis's views agree with contagionism, but this is not so, as Semmelweis himself clearly explains (1861, p. 117):

'Childbed fever is not a contagious disease. A contagious disease is one that produces the contagion by which the disease is spread. This contagion brings about only the same disease in other individuals. ${ }^{6}$ Smallpox is a contagious disease because smallpox generates the contagion that causes smallpox in others. Smallpox causes only smallpox and no other disease. ... Childbed fever is different. This fever can be caused in healthy patients through other diseases. In the first clinic it was caused by a discharging medullary carcinoma of the uterus, by exhalations from a carious knee, and by cadaverous particles from heterogeneous corpses. ... However, childbed fever cannot be transmitted to a healthy maternity patient unless decaying animal-organic matter is conveyed.' 
This puts the point very clearly. A contagious disease is one like smallpox which produces a contagion which infects another patient with the same disease. However puerperal fever, according to Semmelweis, is produced by particles from the corpses of people who did not suffer from puerperal fever, by the discharges of medullary carcinoma of the uterus, and indeed by many other forms of decaying animal-organic matter. That is to say it is produced by things which cannot be specific contagions for puerperal fever. To sum up then. Semmelweis's views as to the causation of puerperal fever contradicted the two dominant theories of disease held at the time - the miasma theory and the contagion theory. It is not so surprising, therefore, given a Kuhnian model of science, that Semmelweis's views were not accepted.

There is even another aspect of the dominant medical paradigm of the time which is contradicted by Semmelweis's ideas about puerperal fever. This is discussed by Codell Carter (1983, pp. 25-28), who points out that Semmelweis differed from his contemporaries on the connected issues of causality and the definition of disease. At the beginning of the $19^{\text {th }}$ century diseases were characterised by their symptoms and increasingly by reference to pathological anatomy. Hence it was perfectly possible for the same disease to have a number of quite different causes. We have seen this in the case of puerperal fever which many doctors thought could be caused by a miasma, or by contagion, or even by the fear of a susceptible patient. ${ }^{7}$ In the second half of the $19^{\text {th }}$ century, however, it became increasingly standard to try to characterise a disease in terms of a single necessary cause. Towards the end of the $19^{\text {th }}$ century, after the development of the germ theory by Pasteur, Lister and Koch, this single necessary cause would characteristically be infection by a particular bacterium. This could often lead to a reclassification of diseases, since two diseases with apparently different symptoms might be seen as the same if they were caused by the same bacterium, while two diseases with similar symptoms might be seen as different if the bacteria involved were different. This new approach which involved defining diseases in terms of their causes had great advantages since it led to more systematic methods of treatment focussed on eliminating the harmful cause. Semmelweis was in fact one of the first to adopt this new approach to causality and the definition of disease, but, the very novelty of the approach must have made it hard for his contemporaries to understand and accept.

In fact Semmelweis's theory of the cause of puerperal fever led him to regard the disease as essentially the same as others which his contemporaries would have regarded as quite different. This comes out in the following passage (Semmelweis, 1861, pp. 11617):

'Suppose we explain childbed fever as a fever of resorption in which the introduction of decaying animal-organic matter leads to disintegration of the blood and exudation. Then childbed fever is not a disease unique to and appearing only in the newly delivered, because as a result of resorption the disease may arise during pregnancy or when giving birth. The disease can be conveyed to infants, whether male or female. In consequence of resorption of decaying matter it can also be found among anatomists, surgeons, in operative cases in surgical wards, etc. Kolletschka also had this disease. Thus childbed fever is not a species of disease; rather it is a type of pyemia.'

Most of Semmelweis's contemporaries would have regarded puerperal fever as a disease 
of the newly delivered mothers and not at all the same as a disease of male surgeons. Semmelweis does indeed try to support his redefinition of puerperal fever by an appeal to pathological anatomy. Thus he writes (1861, p. 77):

'Not only these mothers but also their newborn infants, both male and female, died of childbed fever. I am not alone in speaking of puerperal fever of the newborn. With the exception of the genital areas, the anatomical lesions in the corpses of such newborn infants are the same as the lesions in the corpses of women who die of puerperal fever. To recognize these findings as the consequence of puerperal fever in maternity patients but to deny that identical findings in the corpses of the newborn are the consequence of the same disease is to reject pathological anatomy.'

Codell Carter points out in a footnote to this passage that, although Semmelweis was not alone, he was in the minority. The majority would certainly not have accepted that a newborn male baby could suffer from puerperal fever. For such a child the lesions in the genital areas would, if they existed at all, obviously be different and it would not have been clear why Semmelweis disregarded this fact. Moreover the lesions caused by puerperal fever were in any case rather variable so that to say that the findings in the corpses of the newborn were identical is again somewhat dubious. Only someone like Semmelweis who thought that the underlying cause was the same in the two cases would have seen an identity here.

Although Semmelweis's views diverged very strongly from those of his contemporaries, they are largely compatible with the germ theory of disease. Puerperal fever is now known as 'post-partum sepsis' and is considered to be a bacterial infection. The bacterium principally responsible is streptococcus pyogenes, but other streptococci and staphylococci may be involved. Thus, from a modern point of view, cadaverous particles and other putrid organic matter would not necessarily cause puerperal fever but only if they contain a large enough quantity of living streptococci and staphylococci. However as putrid matter derived from living organisms is a good source of such bacteria, Semmelweis was not far wrong.

It must be remembered, however, that, apart from isolated predecessors, the germ theory of disease only began to take shape following the researches of Pasteur in the late 1850 s and 1860 s. It only became generally accepted in the medical community in the 1880s. All this was in time for Lister, but too late for Semmelweis.

\section{Strengths and Limitations of a Kuhnian Approach}

My claim then is that the principal reason why Semmelweis's theory of the causes of puerperal fever was largely rejected by his contemporaries was that it contradicted the then dominant paradigm in medicine. In other words, Semmelweis experienced difficulties largely because he was a revolutionary scientist. However in section 3 I listed three other possible external reasons for Semmelweis's failure. While not wishing to argue that these were altogether unimportant, I still want to claim that they were of less significance that the Kuhnian reason just given. I will now argue for this thesis.

The first of the external reasons given earlier was that Semmelweis's theory might have been disliked by doctors because it suggested that they themselves were the 
principal cause of puerperal fever by bringing decaying matter to the patients in their examinations. While this sounds plausible, it is, so I believe, unfair to doctors. Doctors of the time were in fact quite used to the idea that they might bring disease to a patient. This was a consequence of the theory of contagion which was part of the dominant paradigm. In Britain doctors favoured the view that puerperal fever was contagious, and consequently there were strong recommendations of measures to prevent the spread of the contagion form one patient to another. For example doctors were advised not only to wash as carefully as possible, but also to change all their clothing completely before attending a delivery. Now, as we know, Semmelweis held that puerperal fever was not a contagious disease, and, as a result of his different view point, he actually criticizes English doctors for taking unnecessary precautions. Thus he says (1861, pp. 149-50):

'For patients in the maternity hospitals the genital region is generally the only area suitable for resorption; thus, in order to contract childbed fever, it is necessary that decaying matter be transmitted to the genital area. Since the clothing of the obstetrician does not contact the genitals, the English custom of changing clothes is a harmless but superfluous measure. ... Clothing can cause childbed fever only when its exhalations enter the uterus with the air, and clothing is not easily contaminated to this degree. Clothing could also cause childbed fever if, for example, the cuff of one's jacket is contaminated with decaying matter and contacts the genitals during the birth. This, however, is not a common happening.'

We see from this that the idea of doctors taking precautions to prevent their passing diseases to patients was quite a standard one of the time. The argument was about what precautions should be taken. Semmelweis's recommendations based on his theory were different from those based on a contagionist approach.

The second external reason concerns Semmelweis's lack of skill in communicating his results to his colleagues. This was indeed a factor in his failure, but again its importance should not be exaggerated. Although Semmelweis did not write up and publish his early results, his friends Hebra and Skoda did so. Moreover Semmelweis says (1861, p. 173): 'My friends and I wrote to the directors of several maternity hospitals.' In this way his views did become known to some of the community. One or two of the directors contacted were quite sympathetic, such as Tilanus of Amsterdam and especially Professor Michaelis of Kiel, one of whose students had studied in the first clinic in Vienna in 1847. Unfortunately Professor Michaelis's acceptance of Semmelweis's doctrine had unfortunate consequences. It convinced him that he had been responsible for the death of his cousin from puerperal fever, and this led him to commit suicide. Semmelweis thus lost someone who could have been an influential supporter.

The majority of those who got to know about Semmelweis's ideas rejected them, and we must examine why they did so. The third external reason given earlier was that Semmelweis had low status both socially and academically because he was a Hungarian in the Austrian empire and a humble assistant rather than a professor. Now such status factors might indeed have had an influence in Vienna, but it is very doubtful whether they would have had any weight outside the Austrian empire. Why should anyone in England or Denmark have cared about whether Semmelweis was Austrian or Hungarian? Moreover scientists do often concern themselves with the content of a paper without even bothering to consider the academic position held by the author - a matter which is likely 
to be of little interest if the author is in a distant land. These considerations will be reinforced if we look at some of the reasons given by those who rejected Semmelweis's ideas.

Simpson of Edinburgh replied somewhat rudely to the letter from Semmelweis and his friends saying that they obviously had not studied the obstetrical literature in English, otherwise they would know that the English had long regarded childbed fever as contagious (Semmelweis, 1861, p. 174). In effect Simpson misinterpreted Semmelweis as saying that childbed fever was a contagious disease. Such misunderstandings of revolutionary ideas are very common among those who are adherents of a dominant paradigm. It is natural for them to reinterpret the statements of the new theory in terms of the concepts of the old paradigm.

Some of Semmelweis's contemporaries did however realise that he was putting forward a radically new conception, and they rejected his approach for precisely that reason. This is shown very clearly in the reaction of Professor Levy of Copenhagen. He learnt about Semmelweis's views form Michaelis of Kiel, and published a detailed criticism of them in 1848. Levy realised that Semmelweis's account of the causes of puerperal fever in effect denied that it was a contagious disease, and it was this that he found implausible. Levy was prepared to admit that those working on the corpses of women who had died of puerperal fever might pass the disease on to patients because the corpses in this case might contain the contagion of puerperal fever. However Levy regarded it as improbable that the disease might be passed on by doctors working on the corpses of those who had died for other reasons. Thus Levy says (quoted in Semmelweis, 1861, p. 182):

'... from the standpoint of contagiousness, if Dr. Semmelweis had limited his opinion regarding infections from corpses to puerperal corpses, I would have been less disposed to denial than I am. ... However, ... , the specific contagium seems to be of little importance to Dr. Semmelweis. ... He is concerned only with general infection from corpses without respect to the disease that led to death. In this respect his opinion seems improbable.'

Levy also produces the following quite convincing argument against Semmelweis (1861, 183):

'... it seems improbable that enough infective infective matter or vapor could be secluded around the fingernails to kill a patient.'

In the later germ theory of disease, this objection could be countered by saying that the infective matter consisted of micro-organisms which could multiply very rapidly within the patient. However the objection was a difficult one for Semmelweis to counter in terms of his own theory. Finally Levy admits that the data do appear to support Semmelweis's theory, but he argues that this could well be just a statistical fluctuation.

Levy's writing shows no sign that he is prejudiced against Semmelweis for any external reason. His article is cool, rational and well-argued. It shows clearly how someone convinced of the dominant medical paradigm of the day would be inclined to reject Semmelweis's new theory as improbable.

So far I have argued for the strengths of a Kuhnian approach, but now I must 
mention some of its limitations. It is true that Kuhn's ideas give a rather convincing explanation of Semmelweis's failure, but it is important to remember that such an explanation is not a justification. ${ }^{8}$ There is no doubt that it would have been better for the patients if the antiseptic practices recommended by Semmelweis had been generally adopted in hospitals. Although Semmelweis's theory contradicted the dominant paradigm regarding the causes of diseases, there was strong statistical evidence in favour of the effectiveness of the procedures he suggested. All this raises doubts about Kuhn's thesis that scientists should not, outside revolutionary periods, consider theories which contradict the dominant paradigm. This thesis may indeed be too dogmatic even for physics, chemistry, and other natural sciences, but it does work quite well in these disciplines. The situation is different for medicine. The Semmelweis case shows in striking fashion that too much respect for the dominant paradigm can damage the interests of patients. I will now try to explain why there is this difference between the natural sciences and medicine.

Kuhn's theory was developed for the natural sciences - principally astronomy (the Copernican revolution), physics (the Einsteinian revolution), and chemistry (Lavoisier's revolution). Medicine, however, differs from a natural science in some fundamental aspects. The aim of medicine is the prevention of diseases whenever this can be done, and the cure of diseases which occur. Its aim is therefore a practical one. To achieve this goal medicine adopts results taken from other sciences, for example X-rays from physics, but it also develops its own specifically medical theories such as the germ theory of disease. By contrast, the aim of any natural science is to discover the truth regarding how nature works in the field under consideration. Naturally of course the discovery of the truth about the natural world generally leads to practical applications. The Copernican theory led to better navigation through the production of better astronomical tables. Lavoisier used his oxygen theory and the discovery that water was composed of hydrogen and oxygen to invent a new method for the industrial production of hydrogen. The method which was developed by him and Meusnier in 1784 was to plunge red-hot iron into water. The gas produced was used for hydrogen balloons which had been invented the year before. Einstein's famous equation $\underline{E}=\underline{m c}^{2}$ shows that mass can be converted into energy. $\underline{\mathrm{c}}$ is the speed of light so that $\underline{\mathrm{c}}^{2}$ is a very large number, and therefore a small quantity of mass can be converted into a very large quantity of energy. This was applied, perhaps unfortunately, in the creation of nuclear arms.

To sum up, the natural sciences seek truth about the world, and the truths discovered often lead to practical applications. Medicine consists above all in practices designed to prevent and cure diseases. Given this, it becomes obvious that any new practice which can be shown statistically to be better than alternative practices as regards either prevention or cure, and which does not have any harmful side-effects, should be adopted even if it is based on a theory which contradicts the dominant paradigm. In fact in such a case, attempts should be made either to explain the success of the new practice using the dominant paradigm, or to modify the dominant paradigm to accommodate the new practice. The adoption of this rule would have led to a better result for Semmelweis's investigations.

This illustrates a particular way in which history of science can influence philosophy of science. Usually historical examples of excellent science are cited in order to exemplify what are argued to be good methodological principles for science. However instances in which the scientific community makes a mistake, as happened in the 
Semmelweis case, can be valuable in suggesting new rules of practice designed to make such mistakes less likely in the future.

\section{Acknowledgements}

I began work on general problems of applying philosophy of science to medicine in the academic year 1999-2000 in connection with preparing some special study modules to be available as options for medical students at King's College London. David Chart was a research fellow at King's during this period to help me with this project, and I am very grateful to him for the numerous and very illuminating discussions we had about these problems. Many of his ideas have, I am sure, found their way into the present paper. At this time I was also working with David Corfield and Jon Williamson on various research projects and I am very grateful for their comments on early versions of this paper. The idea of studying Semmelweis came partly from Zuzana Parusnikova, who used the Semmelweis example in a draft paper on alternative medicine which she kindly sent me to read. Earlier versions of the paper were read at the philosophy department's seminar in King's and at my presidential address to the British Society for the Philosophy of Science in June 2003. I gave an Italian version of this talk to meetings at Bologna, Rome and Palermo. I am very grateful for the comments I received on these occasions, as well as for comments from Rachel Cooper, Ladislav Kvasz, and an anonymous referee. Some of these comments led to extensive rewriting. I would also like to thank the AHRB for providing a term of sabbatical leave in the spring of 2003 during which I was able to work on this paper. 


\section{Notes}

1. In describing Semmelweis's investigation, I will largely follow Semmelweis's own account in his 1861, but I have also found Codell Carter (1983) and Sinclair (1909) helpful.

2. The account of Kuhn which follows will be based on his original ideas as set out in his 1962, and will not involve subsequent changes made by Kuhn which were, in my opinion, mainly changes for the worse.

3. It is possible that Hempel in his later years might not have been averse to changing his analysis to include Kuhnian elements. In fact in an interview conducted in 1982, Hempel said the following about Kuhn whom he first met in 1963: 'I was very much struck by his ideas. At first I found them strange and I had very great resistance to these ideas, his historicist, pragmatist approach to problems in the methodology of science, but I have changed my mind considerably about this since then.' This quotation appears on p. 115 of Wolters (2003), an article which contains an interesting account of Hempel's later philosophy.

4. This theory was thought to apply to the majority of peptic ulcers. There are some peptic ulcers which have other causes, e.g. the use of NSAIDs (nonsteroidal antiinflammatory drugs) such as aspirin or ibuprofen. However these cases will not be considered in what follows. 
5. The reclassification involved in this case is also stressed by Thagard. See his interesting remarks on this subject in the last paragraph of his 1999, p. 51.

6. Semmelweis is here using the standard definition of contagion as is shown by considering what Henle has to say in his 1840. Henle writes (p. 954): 'The academicians ... say "... The contagious agent ... is a material which is formed by a disease and which occasions the same disease in others." Henle himself uses almost the same definition of contagion as that which he attributes to the 'academicians'. He says (p. 916): 'The contagious agent is a substance which in the course of a disease is excreted by the sick organism ... and which communicated to healthy individuals, produces the same disease in them.'

7. Henle also considers it possible that the same disease can be produced by mental as well as physical causes. Thus he attributes ague, i.e. malaria, to a miasma, but goes on to say (1840, p. 962): 'It must be added, that other influences besides the marsh miasma, for instance, constant mental sufferings, also appear to give rise to ague.'

8. This point was put to me forcibly by Jon Williamson who consequently inspired the ideas of the remaining part of this section

\section{References}

Codell Carter, K. (1983). Translator's Introduction to Semmelweis (1861), pp. 3-58.

Godlee, Sir Rickman (1917). Lord Lister. London: Macmillan.

Hempel, C.G. (1966). Philosophy of Natural Science. Englewood Cliffs, N.J.: PrenticeHall.

Henle, J. (1840). On Miasmata and Contagia. English Translation in Bulletin of the History of Medicine, 1938, 6, 911-983.

Kuhn. T.S. (1962). The Structure of Scientific Revolutions. Chicago \& London: University of Chicago Press.

Lipton, P. (1991). Inference to the Best Explanation. (Paperback Edition) London and New York: Routledge.

Loudon, I. (1992). Death in Childbirth. Oxford: Clarendon Press.

Porter, R. (1997). The Greatest Benefit to Mankind. A Medical History of Humanity from Antiquity to the Present. London: HarperCollins.

Semmelweis, I. (1861). The Etiology, Concept, and Prophylaxis of Childbed Fever. 
1983 English Translation by K. Codell Carter. Wisconsin: The University of Wisconsin Press.

Sinclair, Sir William J. (1909). Semmelweis. His Life and his Doctrine. Manchester: Manchester University Press.

Thagard, P. (1999). How Scientists Explain Disease. Pinceton, New Jersey: Princeton University Press.

Wolters, G. (2003). Carl Gustav Hempel. Pragmatic Empiricist. In P. Parrini, W. C. Salmon, M. H. Salmon (Eds.), Logical Empiricism: Historical \& contemporary Perspectives (pp. 109-122), Pittsburgh: University of Pittsburgh Press.

TABLE 1

Death Rates in the First and Second Clinics of the Vienna Maternity Hospital for the years $1833-1858$

First Clinic

\begin{tabular}{|c|c|c|c|c|c|c|}
\hline & & \\
\hline & Births & Deaths & Rate $(\%)$ & Births & Deaths & Rate (\%) \\
\hline 1833 & 3,737 & 197 & \multirow{9}{*}{$\begin{array}{l}5.27 \\
7.72\end{array}$} & \multirow{9}{*}{$\begin{array}{r}353 \\
1,744 \\
1,682 \\
1.670 \\
1,784 \\
1,779 \\
2,010 \\
2,073 \\
13,095 \\
\end{array}$} & \multirow{2}{*}{$\begin{array}{c}8 \\
150\end{array}$} & \multirow{2}{*}{2.27} \\
\hline 1834 & 2,657 & 205 & & & & \\
\hline 1835 & 2,573 & 143 & & & 84 & 4.99 \\
\hline 1836 & 2,677 & 200 & & & 131 & 7.84 \\
\hline 1837 & 2,765 & 251 & & & 124 & 6.95 \\
\hline 1838 & 2,987 & 91 & & & 88 & 4.95 \\
\hline 1839 & 2,781 & 151 & & & 91 & 4.53 \\
\hline 1840 & 2,889 & 267 & & & 55 & 2.65 \\
\hline Total & 23,066 & 1,505 & & & \multirow[t]{2}{*}{731} & \\
\hline \multicolumn{3}{|c|}{$\begin{array}{c}\text { LJ, } \\
\text { Average 1833-1840 }\end{array}$} & 6.53 & & & 5.58 \\
\hline 1841 & 3,036 & 237 & 7.8 & \multirow{8}{*}{$\begin{array}{r}2,442 \\
2,659 \\
2,739 \\
2,956 \\
3,241 \\
3,754 \\
17,791 \\
\end{array}$} & 86 & 3.5 \\
\hline 1842 & 3,287 & 518 & 15.8 & & 202 & 7.6 \\
\hline 1843 & 3,060 & 274 & 9.0 & & 164 & 6.0 \\
\hline 1844 & 3,157 & 260 & 8.2 & & 68 & 2.3 \\
\hline 1845 & 3,492 & 241 & 6.9 & & 66 & 2.04 \\
\hline 1846 & 4,010 & 459 & 11.5 & & 105 & 2.8 \\
\hline Total & \multirow{2}{*}{\multicolumn{2}{|c|}{$\begin{array}{cc}20,042 & 1,989 \\
2 \text { eraoe } 1841-1846\end{array}$}} & & & \multirow[t]{2}{*}{691} & \\
\hline & & & 9.92 & & & 3.88 \\
\hline
\end{tabular}




\begin{tabular}{|c|c|c|c|c|c|c|}
\hline 1847 & 3,490 & 176 & \multirow{13}{*}{$\begin{array}{l}5.04 \\
1.27 \\
2.67 \\
1.98 \\
1.79 \\
4.05 \\
2.23 \\
9.11 \\
5.41 \\
3.98 \\
2.94 \\
2.05\end{array}$} & \multirow{13}{*}{$\begin{array}{r}3,306 \\
3,319 \\
3,371 \\
3,261 \\
3,395 \\
3,360 \\
3,480 \\
3,396 \\
2,938 \\
3,070 \\
3,795 \\
4,179 \\
40,870\end{array}$} & \multirow{2}{*}{$\begin{array}{l}32 \\
43\end{array}$} & \multirow{2}{*}{$\begin{array}{l}0.97 \\
1.30\end{array}$} \\
\hline 1848 & 3,556 & 45 & & & & \\
\hline 1849 & 3,858 & 103 & & & 87 & 2.58 \\
\hline 1850 & 3,745 & 74 & & & 54 & 1.66 \\
\hline 1851 & 4,194 & 75 & & & 121 & 3.56 \\
\hline 1852 & 4,471 & 181 & & & 192 & 5.71 \\
\hline 1853 & 4,221 & 94 & & & 67 & 1.93 \\
\hline 1854 & 4,393 & 400 & & & 210 & 6.18 \\
\hline 1855 & 3,659 & 198 & & & 174 & 5.92 \\
\hline 1856 & 3,925 & 156 & & & 125 & 4.07 \\
\hline 1857 & 4,220 & 124 & & & 83 & 2.19 \\
\hline 1858 & 4,203 & 86 & & & 60 & 1.44 \\
\hline \multirow[t]{2}{*}{ Total } & 47,935 & 1,712 & & & 1,248 & \\
\hline & \multicolumn{2}{|c|}{ Average 1847-1858 } & 3.57 & & & 3.05 \\
\hline
\end{tabular}

Source: Semmelweis, 1861, Tables 1, 11, and 12. Arithmetic does not seem to have been Semmelweis's strong point, and many of the arithmetical calculations in his tables are incorrect. I have assumed that the basic figures for births and deaths are accurate, and have corrected the calculated totals and percentages where they are wrong. Semmelweis's mistakes are for the most part rounding-up errors altering only the last decimal place. No mistake is sufficiently great to affect his argument. 


\section{TABLE 2}

Death Rates in the First Clinic of the Vienna Maternity Hospital for the year 1847 on a monthly basis

\begin{tabular}{|c|c|c|c|}
\hline 1847 & Births & Deaths & Rate $(\%)$ \\
\hline January & 311 & 10 & 3.22 \\
\hline February & 312 & 6 & 1.92 \\
\hline March & 305 & 11 & 3.61 \\
\hline April & 312 & 57 & 18.27 \\
\hline May & 294 & 36 & 12.25 \\
\hline Total & 1,534 & 120 & \\
\hline \multicolumn{3}{|c|}{ Average January to May } & 7.82 \\
\hline June & 268 & 6 & 2.24 \\
\hline July & 250 & 3 & 1.20 \\
\hline August & 264 & 5 & 1.89 \\
\hline September & 262 & 12 & 4.58 \\
\hline October & 278 & 11 & 3.96 \\
\hline November & 246 & 11 & 4.47 \\
\hline December & 273 & 8 & 2.93 \\
\hline Total & 1,841 & 56 & \\
\hline \multicolumn{3}{|c|}{ Average June to December } & 3.04 \\
\hline
\end{tabular}

Average January to March $=2.91 \%$

Average April to May $=15.35 \%$

Source: Semmelweis, 1861, Tables 5 and 6. I have corrected Semmelweis's errors of calculation as in Table 1 except for the month of February. Here the number of births is originally given as 912 and the rate as $1.92 \%$. I have preferred in this instance to change 
the number of births to 312 (assuming 9 was a misprint for 3 ), and retain the rate at $1.92 \%$. The figure of 912 is obviously out of line with the others and must be mistaken. Even with this alteration the total number of births comes to 3,375 which differs from the figure of 3,490 given in table 1 (though the number of deaths in the two tables agrees). One can only conclude that Semmelweis's statistics, though they give a correct general impression, are not to be trusted in detail. 